\title{
Preparation and Characterization of Hydrophilic Wetting-Modified Polyamide Fibers
}

\author{
Liang Li, ${ }^{1,2,3}$ Shuping Liu, ${ }^{1,2,3}$ Rangtong Liu $\mathbb{D}^{1,2,3}$ Changjun Geng, ${ }^{1,2,3}$ and Zedong Hu ${ }^{1,2,3}$ \\ ${ }^{1}$ Zhongyuan University of Technology, 41 Zhongyuan Road (M), Zhengzhou, Henan Province, China \\ ${ }^{2}$ Collaborative Innovation Center of Textile and Clothing in Henan Province, 41 Zhongyuan Road (M), Zhengzhou, China \\ ${ }^{3}$ Henan Provincial Key Laboratory of Functional Textile Materials, 41 Zhongyuan Road (M), Zhengzhou, Henan Province, China
}

Correspondence should be addressed to Rangtong Liu; ranton@126.com

Received 8 September 2019; Revised 9 January 2020; Accepted 26 February 2020; Published 13 April 2020

Academic Editor: Mona Semsarilar

Copyright (C) 2020 Liang Li et al. This is an open access article distributed under the Creative Commons Attribution License, which permits unrestricted use, distribution, and reproduction in any medium, provided the original work is properly cited.

\begin{abstract}
To improve the moisture absorption and air permeability of polyamide (PA) fibers, the modified fibers with porous structure were fabricated by melt spinning using polyethylene glycol (PEG) as the pore-forming agent due to its high solubility in water. The effects of pores caused by different PEG contents on the structure and properties of modified fibers were analyzed by electron microscopy (SEM), Fourier transform infrared spectroscopy (FTIR), X-ray diffraction (XRD), and water contact angle. These results indicate that the porosity of modified fibers increases with PEG content increasing. Moreover, the formation of pores obviously affects the crystal forms of modified fibers. The alpha crystal gradually decreases and the gamma crystal increases with the porosity increasing. Furthermore, with respect to the modified fibers obtained from $47.33 \%$ PEG content, its water absorption increases from $3.78 \%$ of pure PA to $19.76 \%$ and its contact angle decreases from $116^{\circ}$ to $85^{\circ}$. In addition, due to the interaction of hydrogen bond occurring between PA and PEG during the spinning process, the elongation at break, rupture work, and initial modulus of modified fibers were improved when the porosity was not more than $4.23 \%$.
\end{abstract}

\section{Introduction}

The polyamide (PA) is an important polymeric material and widely applied from industrial areas to civil fields, especially sportswear, because of its light weight, softness, high elastic resilience, good wear resistance, excellent chemical resistance and mechanical properties. Nowadays, it has become the largest synthetic fiber after the polyester in the clothing industry. However, due to its poor moisture absorption and air permeability, the property of wearing comfort is not excellent. To solve this problem, there are many modified methods executed such as blending modification [1-3], copolymerization modification [4-6], surface modification [7-9], special-section modification $[10,11]$ and postfinishing [12-14]. For example, Pappas et al. [15] functionalized the PA surface by the addition of new reactive chemical groups to improve its wettability. Miao et al. [16] found that the rate of oil and water absorption all decreased significantly after the treatment by an ionized mixture of gases for nylon fabric, which was well-agreed with the research of Samanta K K team [17]. Generally speaking, there exist advantages and disadvantages for each method. As for the copolymerization modification, its complex process increases production difficulty and cost. The surface modification, especially plasma treatment, is much complicated and difficult to industrialize. With respect to the special-section modification, the manufacture of the spinneret equipment is complex and requires high precision. There exists poor durability for the postfinishing modification. Comparing with the above mentioned methods, the blending modification is relatively popular and simple, thus, it is usually applied to prepare the modified fibers in chemical fiber industry. Moreover, there are many literatures on PA blending modification [18-20].

In recent years, with the further application of the ultrafiltration membrane, the phase inversion method through adding suitable additives is increasingly paid attention to 
improve membrane performances and many additives can be employed [21-23]. The polyethylene glycol (PEG), which is nontoxic, nonirritant and highly soluble in water, is usually used as a polymeric additive to enhance the pore connectivity and permeability of membranes during the fabrication of solution casting. Yang et al. [24] blended PEG into polystyrene-block-poly (acrylic acid) to adjust the pore size and permeability of isoporous membranes. They found that when the membrane permeability reached the largest, there existed a balance between the micro- and macrophase separation. Some researchers investigated the effects of PEG with different molecular weights on the membrane formation and water permeation $[25,26]$. In view of this, the hydrophilic wetting PA fibers can be obtained by the phase inversion method through nonsolvent additives.

To our best knowledge, although this phase inversion method through adding some additives to enhance the pore connectivity and permeability of materials was usually applied in membrane fabrication, there were relatively few literatures to improve the hydrophilic wettability and air permeability of PA fibers. An attempt was made to investigate the feasibility using PEG as the pore-forming agent to fabricate modified fibers with pore structure by melt spinning. The amount of PEG on the porosity was discussed, and the effects of porosity on the structure and performances of modified fibers were characterized.

\section{Experimental}

2.1. Materials. Polyamide 6 (PA6, technical grade) was purchased from Kunshan Hongyue Plastic Co., Ltd (Suzhou, China). PEG 2000 (analysis grade) and potassium bromide (spectrum grade) were provided by Tianjin Kemiou Chemical Reagent Co., Ltd (Tianjin, China). Glycerol (analysis grade) was bought from Tianjing Fengchuan Chemical Reagent Technologies Co., Ltd (Tianjing, China).

2.2. Preparation of Modified Fibers. The blending method of melt spinning was employed to obtain modified fibers. The schematic diagram shown in Figure 1 mainly consisted of the following steps.

Mixture preparation: firstly, PEG 2000 was melted in a water bath at $90^{\circ} \mathrm{C}$. Then granular PA was added and stirred by a mechanical stirrer for $5 \mathrm{~h}$. In order to research the effects of the porosity on the structure and properties of modified fibers, a series of PA/PEG blends was prepared with a PEG content of $0 \%, 8.85 \%, 18.79 \%, 28.25 \%, 38.66 \%$ and $47.33 \%$, respectively.

Blended fibers preparation: the blends were melt-spun into fibers by a single screw spinning machine at $235^{\circ} \mathrm{C}-285^{\circ} \mathrm{C}$ with a winding speed of $1 \mathrm{~m} / \mathrm{min}$.

Modified fibers preparation: the melt-spun fibers by PA and PEG were immersed into distilled water at room temperature to remove the PEG which was highly solule in water. Meanwhile, to ensure complete removal of PEG, the sample was taken out at a regular interval $(30 \mathrm{~min})$ and weighted after drying. The modified fibers were obtained when the mass no longer changed after weighing 5 times

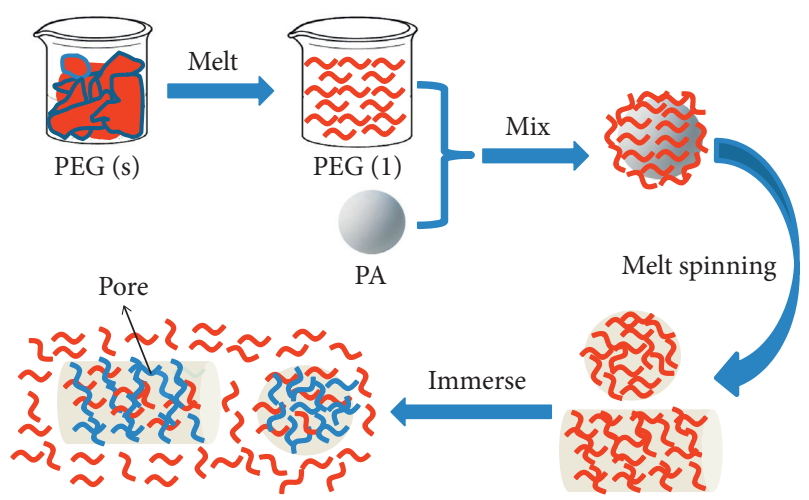

Figure 1: Schematic diagram of the preparation process of modified fibers.

continuously. Moreover, the porosity of modified fibers depended on the PEG content during the preparation of modified fibers. In general, high PEG content corresponded to large porosity in fibers.

\section{Characterization}

3.1. Scanning Electron Microscopy. The surface and crosssection morphologies of modified fibers were observed by a scanning electron microscope (SEM, SIGMA500, Carl Zeiss Jena, Germany). The specimens were sputtered with thin gold to avoid electron-charging effects before SEM observation.

3.2. Infrared Spectroscopy Analysis. The chemical structures of modified fibers were characterized by an infrared spectrometer (TENSOR37, Bruker Optics, Germany) using the $\mathrm{KBr}$ discs method. The spectra were collected in the wavenumber region of $400-4000 \mathrm{~cm}^{-1}$ with a spectral resolution of $4 \mathrm{~cm}^{-1}$.

3.3. X-Ray Analysis. X-ray analysis was conducted by an X-ray diffractometer (Ultima-Iv, Rigaku Corporation, Japan) with $\mathrm{CuKa}$ radiation to measure the crystal structure of modified fibers. The fiber samples were cut into pieces and the $1.5 \mathrm{~cm} \times 1.5 \mathrm{~cm}$ thin sheet was pressed before testing. The step-scan method with $10^{\circ} / \mathrm{min}$ scanning rate was performed to determine the changes of fibers' crystalline structure.

3.4. Porosity Calculation. A certain amount of modified fibers was weighed $\left(W_{\text {air }}\right)$ and then immersed into glycerol for $1 \mathrm{~h}$ to absorb sufficiently. After that, it was taken from the glycerol and the surface was dried using oil-absorbing sheets. At last, its quality was weighed again $\left(W_{G}\right)$. The porosity $(P)$ was calculated according to the following formula.

$$
P=\frac{\rho_{\mathrm{PA}}\left(W_{G}-W_{\text {air }}\right)}{\rho_{\mathrm{PA}}\left(W_{G}-W_{\text {air }}\right)+W_{\text {air }} \rho_{G}-W_{G} \rho_{\text {air }}} \times 100 \%,
$$

where $W_{\text {air }}$ and $W_{G}$ are the quality of modified fibers before and after glycerol absorption. The $\rho_{\text {air }}\left(1.293 \times 10^{-3} \mathrm{~g} / \mathrm{cm}^{3}\right), \rho_{G}$ 


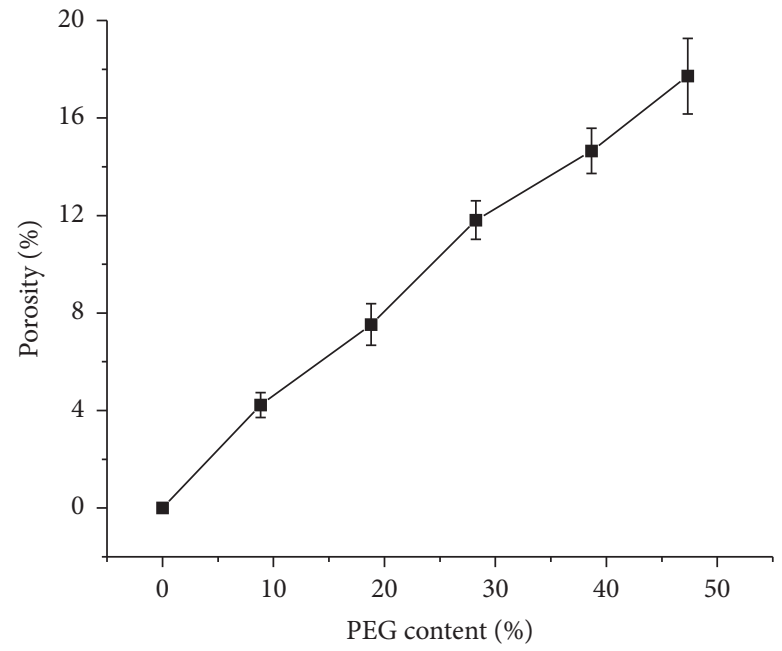

Figure 2: Porosity of modified fibers obtained from different PEG contents.
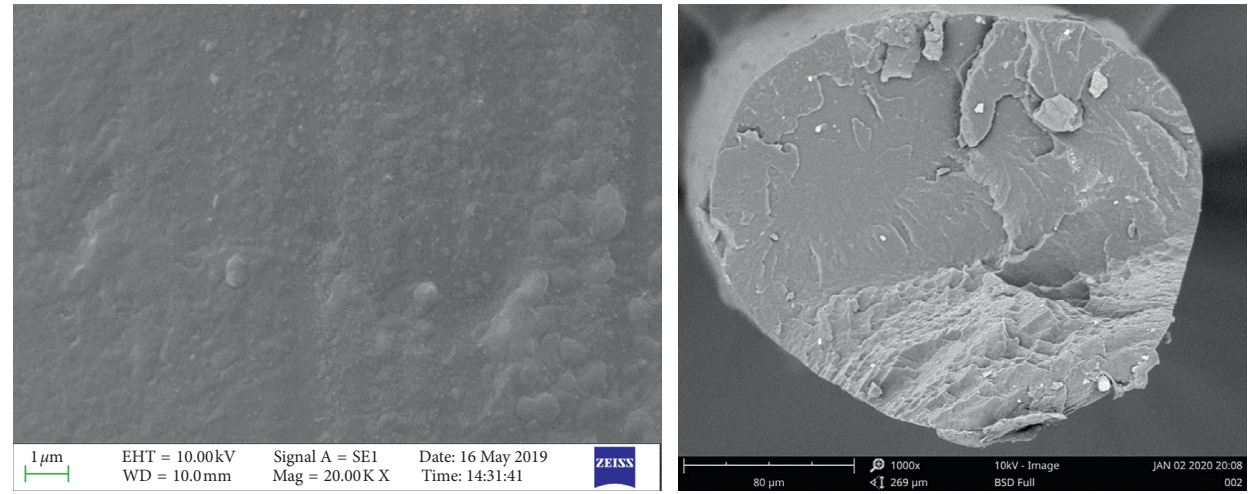

(a)
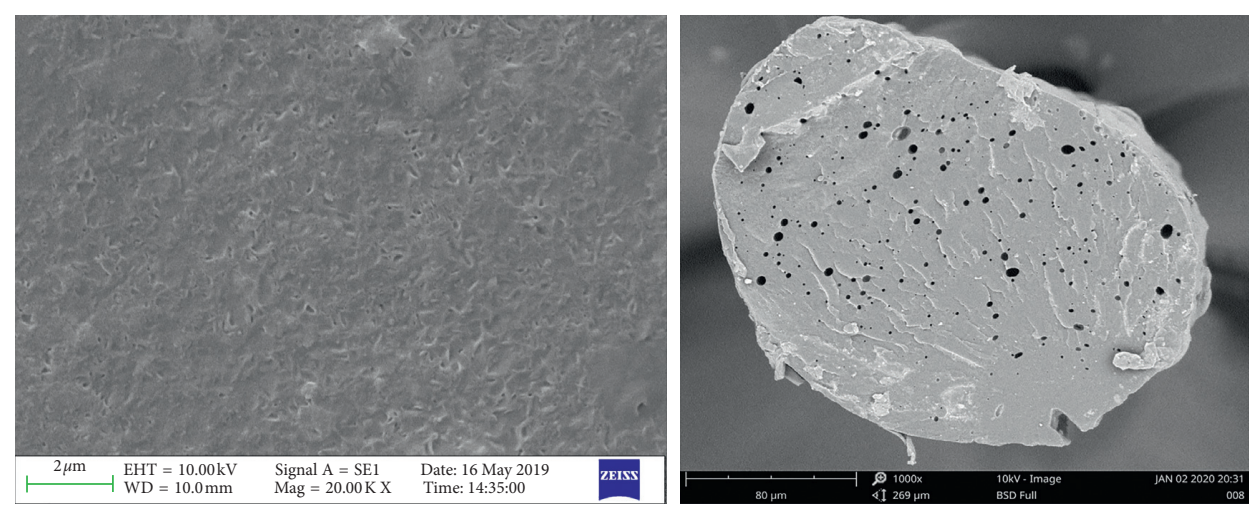

(b)

FIgURE 3: Surface and cross-section SEM images of modified fiber with $11.81 \%$ porosity and pure PA fiber.(a) Pure PA fiber. (b) Modified fiber.

$\left(1.2617 \mathrm{~g} / \mathrm{cm}^{3}\right)$ and $\rho_{\mathrm{PA}}\left(1.14 \mathrm{~g} / \mathrm{cm}^{3}\right)$ were the densities of air, glycerol and PA, respectively.

3.5. Water Absorption. The calculation of water absorption was very similar to that of porosity and just the glycerol was replaced by water. Firstly, the quality of modified fibers after PEG removal was weighed $\left(W_{1}\right)$. Then they were immersed into water to fully absorb and weighed again after the water on the fiber surface was removed $\left(W_{2}\right)$. The water absorption $(\mathrm{Wa})$ was calculated by the following formula.

$$
W a=\frac{W_{2}-W_{1}}{W_{1}} \times 100 \% .
$$

3.6. Wettability. The wettability of modified fibers was measured by the water contact angle measurement with a contact angle meter (OCA2, Dataphysics instruments gmbh, 


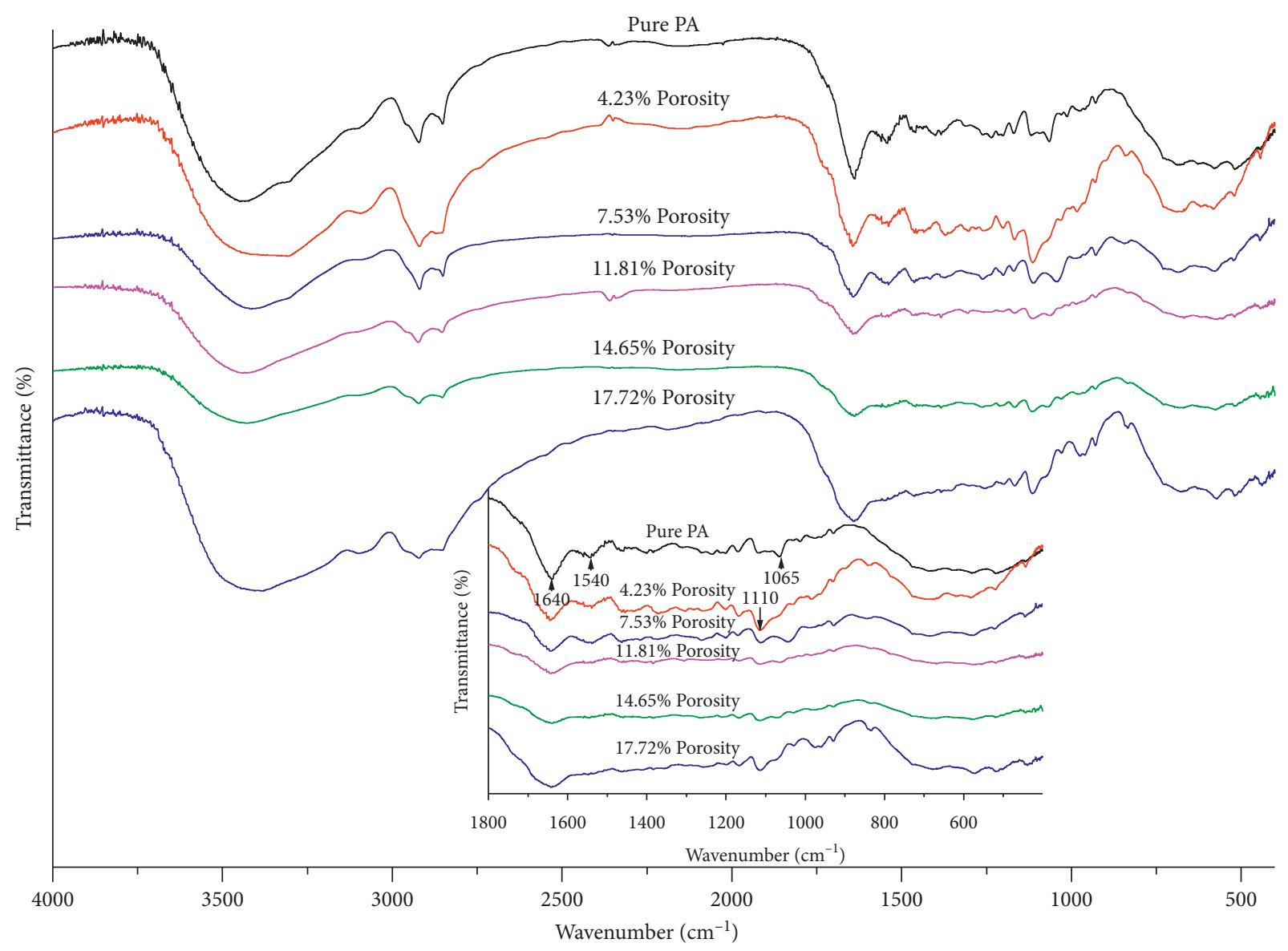

FIgURE 4: FTIR spectra of modified fibers with different porosities.

Germany) using the suspension drop method and the injection volume of water was $0.5 \mu \mathrm{ml}$. The fibers should be closely aligned in a plane before the testing and the water contact angle was the angle of water droplets reaching equilibrium.

3.7. Physical Properties. The fineness of modified fibers was measured by a XD-1 fiber fineness machine made by Donghua University (Shanghai, China). A $20 \mathrm{~mm}$ gauge length was employed and 20 tests were carried out for each sample to estimate the average fineness. Mechanical properties of modified fibers were determined by LILY-06ED fiber tensile tester. The gauge length was $20 \mathrm{~mm}$ and tensile speed was $20 \mathrm{~mm} / \mathrm{min}$. A minimum of 20 tests were carried out for each sample to estimate the average value.

\section{Results and Discussion}

4.1. Porosity of Fibers. The porosity of modified fibers depended on the PEG content and calculated by the formula (1). Figure 2 plotted the porosity of modified fibers obtained from different PEG contents. It indicated that the porosity of modified fibers increased significantly with the increase of PEG content, however, it was much smaller than the blending ratio of PEG. Two possible reasons were that one was the incomplete removal of PEG during the immersion process causing by the interaction between PEG and PA during the spinning. The other was the mass loss of PEG during the spinning for its low melting point.

4.2. Surface and Cross-Section Morphologies of Fibers. The surface and cross section of modified fibers were affected by the pores caused by the PEG removal. Figure 3 showed the surface and cross-section SEM images of modified fibers with $11.81 \%$ porosity and pure PA. It demonstrated that the surface and cross section of pure PA fibers were smooth and compact. With respect to the modified fibers, the surface and cross section presented many irregular pores which were resulted from the PEG removal after they were immersed in water.

4.3. Chemical Structure of Fibers. Infrared spectra are very sensitive to the chemical structure of materials. Figure 4 presented the infrared spectra of PA fibers with different porosities. It demonstrated that there were prominent peaks at $3430,2920,2850$ and $1640 \mathrm{~cm}^{-1}$ for all fibers, which were the characteristic absorption peaks of PA6 [27]. The strong absorption peak centered at $3430 \mathrm{~cm}^{-1}$ was attributed to the $\mathrm{N}-\mathrm{H}$ stretching vibration of amide. The absorption peaks located at $2920 \mathrm{~cm}^{-1}$ and $2850 \mathrm{~cm}^{-1}$ were related to the antisymmetric and symmetric stretching modes of 
methylene, respectively. The strong absorption peak at $1640 \mathrm{~cm}^{-1}$ was associated with the bending vibration of amide I and water [28]. The intensity of these absorption peaks decreased gradually with the porosity increasing except for that of the modified fiber with $17.72 \%$ porosity. Moreover, the intensities of absorption peaks located at $1540 \mathrm{~cm}^{-1}$ and $1065 \mathrm{~cm}^{-1}$, which were caused by amide II and $\mathrm{C}-\mathrm{N}$ stretching vibration, decreased gradually and until disappeared with the increase of the porosity. In addition, the characteristic absorption peaks of PEG resulting from the $\mathrm{C}-\mathrm{H}$ bending vibration of methylene and $\mathrm{C}-\mathrm{O}-\mathrm{C}$ symmetric stretching vibration appeared at $1380 \mathrm{~cm}^{-1}$ and $1110 \mathrm{~cm}^{-1}$ in modified fibers $[29,30]$. The changes of FTIR spectra indicated that there were some reactions occurred between PA and PEG during spinning which led to a little amount of PEG incomplete removal [31]. When the PEG content reached $47.33 \%$, there were more pores in modified fibers and these pores easily absorbed the moisture in the air which resulted in the enhancement of absorption peaks intensity at $3430 \mathrm{~cm}^{-1}$ and $1640 \mathrm{~cm}^{-1}$.

4.4. Crystal Structure of Fibers. PA is a highly crystalline polymer with various crystalline forms. The existence of pores and a little PEG affected the crystalline structure of modified fibers. The XRD fitting diagram of pure PA fibers was given in Figure 5. There were three crystallization peaks for pure PA fibers which were alpha crystallization peaks located at $20.9^{\circ}$ and $23.11^{\circ}$ (peak 1 and 4 as shown in Figure 5) and gamma crystallization peak at $21.35^{\circ}$ (peak 2 in Figure 5), as well as an amorphous peak (peak 3 in Figure 5), respectively $[32,33]$. As shown in Figure 6, the XRD patterns of modified fibers changed significantly and the three crystallization peaks separated obviously because of the formation of pores. Moreover, the intensity of the alpha crystallization peak at $20.9^{\circ}$ decreased gradually and the intensity located at $23.11^{\circ}$ increased with the increase of the porosity, however, the gamma crystallization peak firstly increased and then decreased, which demonstrated that the existence of pores obviously changed the crystal form and crystal face structures of PA fibers. In addition, although the pure PEG was also a highly crystalline material, there was no the characteristic diffraction peak in XRD patterns. It indicated that the PEG did not form an independent crystalline phase in modified fibers. The reason was that the relatively little amount of PEG could not crystallize, and, furthermore, the reactions between PEG and PA resulted in the absence of free PEG molecular.

4.5. Hydrophilic Wettability of Fibers. The porous structure affected the hydrophilic wettability of fibers which was characterized by the water absorption and contact angle. The water absorption and contact angle of modified fibers with different porosities were shown in Figures 7 and 8 . With the porosity increasing, the water absorption increased continuously, and the contact angle decreased gradually. Moreover, when the porosity reached $14.65 \%$, the modified fibers had changed from hydrophobic to hydrophilic. The reason was that the roughness surface of modified fibers

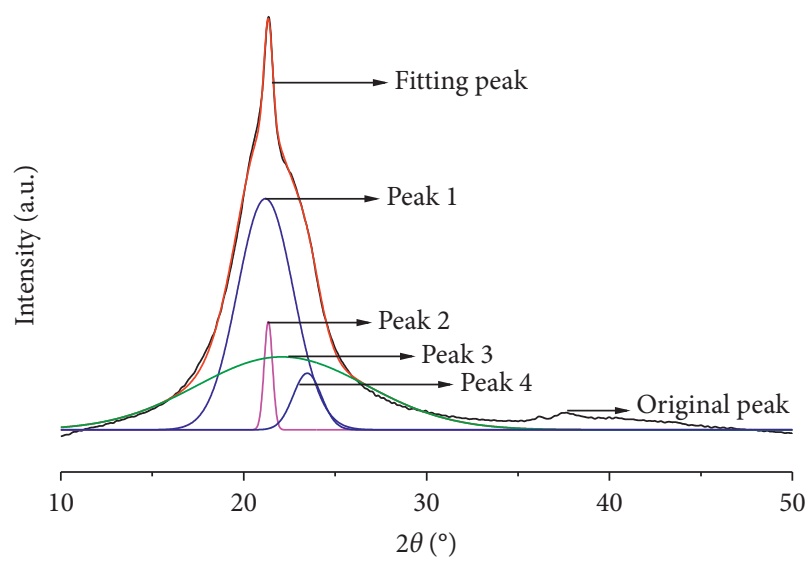

FIGURE 5: XRD fitting diagram of pure PA fiber.

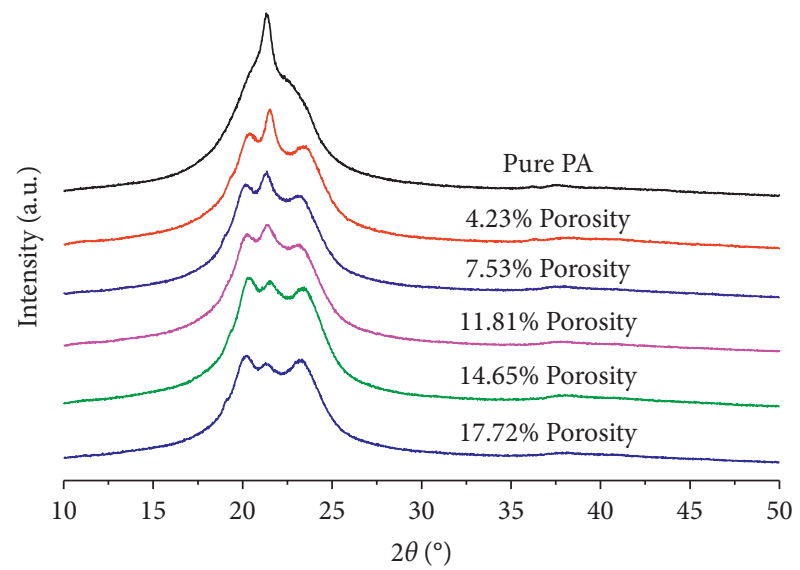

FIGURE 6: XRD patterns of modified fibers with different porosities.

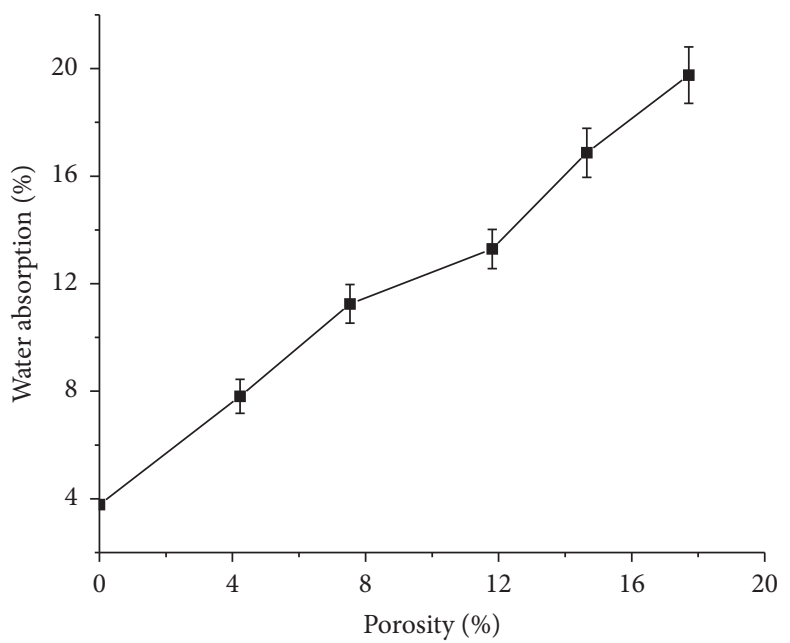

Figure 7: Water absorption of modified fibers with different porosities.

caused by pore structure was conducive to improve the hydrophilic wettability of fibers.

4.6. Physical and Mechanical Performances of Fibers. The physical and mechanical properties of modified fibers with different porosities were are listed in Table 1. The fineness 


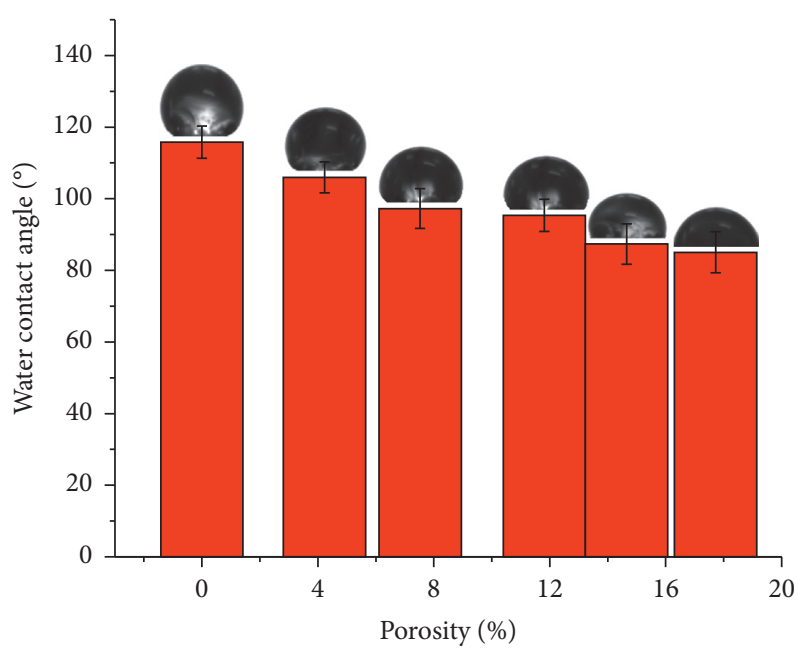

FIGURE 8: Water contact angle of modified fibers with different porosities.

Table 1: Parameters of physical and mechanical performances of PA fibers with different porosities.

\begin{tabular}{lccccc}
\hline Porosity (\%) & Fineness (dtex) & Tensile strength (cN/dtex) & Elongation at break (\%) & Rupture work (cN·mm) & Initial modulus (cN/dtex) \\
\hline 0 & 8.77 & 11.77 & 228.98 & 2580.75 & 123.38 \\
4.23 & 8.47 & 11.61 & 233.81 & 3621.47 & 126.68 \\
7.53 & 8.05 & 10.03 & 231.22 & 2420.12 & 120.17 \\
11.81 & 7.67 & 9.92 & 225.36 & 1806.42 & 104.43 \\
14.65 & 7.41 & 9.61 & 221.51 & 1553.71 & 96.02 \\
17.72 & 6.97 & 8.69 & 198.87 & 1524.89 & 94.08 \\
\hline
\end{tabular}

and tensile strength of modified fibers decreased gradually with the porosity increasing; however, the elongation at break, rupture work and initial modulus appeared firstly increasing and then decreasing. The main reason for the increase was that a little amount of PEG played the plasticizing and toughening roles in fibers. In addition, once the porosity exceeded $4.23 \%$, the properties of modified fibers began to deteriorate for the pore increase in fibers leading to the slip and fracture of the molecular chains during the stretching process.

\section{Conclusion}

The purpose of this paper was to investigate the effects of the porosity caused by different PEG contents on the performances of PA modified fibers. The results demonstrated that the performances of modified fibers were obviously affected by the porous structure. Due to the existence of pores, the morphologies of surface and cross section and crystal forms of modified fiber distinctly changed. Moreover, the hydrophilic wettability of modified PA fibers increased with the porosity increasing. The modified fibers could be completely transformed into hydrophilic fibers when the porosity was large enough. With the porosity increasing, the fineness and tensile strength of modified fibers decreased gradually, however, the elongation at break, rupture work and initial modulus appeared firstly increasing and then decreasing because of a small amount of PEG residues and pores. When the porosity was not more than $4.23 \%$, the modified fibers with excellent mechanical properties and hydrophilic wettability were capable to be obtained.

\section{Data Availability}

Data are available from the corresponding author upon reasonable request.

\section{Conflicts of Interest}

The authors declare that they have no conflicts of interest.

\section{Acknowledgments}

This work was supported by National Key R\&D Program of China (grant nos. 2017YFB0309100, 2017), Henan Provincial Collaborative Innovation Center of Textile and Clothing, and Henan Provincial Key Laboratory of Functional Textile Materials, China.

\section{References}

[1] J. S. Jeong, S. Y. Jeon, T. Y. Lee et al., "Fabrication of MWNTs/ nylon conductive composite nanofibers by electrospinning," Diamond and Related Materials, vol. 15, no. 11-12, pp. 1839-1843, 2006.

[2] D. Khaparde, "Preparation and prediction of physical properties of cellulose acetate and polyamide polymer blend," Carbohydrate Polymers, vol. 173, pp. 338-343, 2017.

[3] X. Yu, X. Wang, Z. Zhang, S. Peng, H. Chen, and X. Zhao, "High-performance fully bio-based poly (lactic acid)/polyamide11 (PLA/PA11) blends by reactive blending with multifunctionalized epoxy," Polymer Testing, vol. 78, p. 105980, 2019. 
[4] C. Makhlouf, S. Marais, and S. Roudesli, "Graft copolymerization of acrylic acid onto polyamide fibers," Applied Surface Science, vol. 253, no. 12, pp. 5521-5528, 2007.

[5] J. Ma, "Preparation and properties of hydrophilic antistatic polyester fiber," Tianjin Polytechnic University, Tianjin, China, M.S. thesis. pp. 5-10, 2017.

[6] Q. Wang and F. Y.D. Zhao, "Hydrophilic-lipophilic modification of PET/PA6 bicomponent spunbonded spunlaced nonwovens by ultraviolet grafting," Journal of Textile Research, vol. 36, no. 11, p. 99, 2015.

[7] L. Zhu, C. Wang, and Y. Qiu, "Influence of the amount of absorbed moisture in nylon fibers on atmospheric pressure plasma processing," Surface and Coatings Technology, vol. 201, no. 16-17, pp. 7453-7461, 2007.

[8] M. Parvinzadeh, R. Assefipour, and A. Kiumarsi, "Biohydrolysis of nylon 6, 6 fiber with different proteolytic enzymes," Polymer Degradation and Stability, vol. 94, no. 8, pp. 1197-1205, 2009.

[9] M. Gul Dincmen, P. J. Hauser, and N. C. Gursoy, "Plasma induced graft polymerization of three hydrophilic monomers on nylon 6, 6 fabrics for enhancing antistatic property," Plasma Chemistry and Plasma Processing, vol. 36, no. 5, pp. 1377-1391, 2016.

[10] G. B. Zhao, H. T. Li, Y. Y. Huang, and X. H. Guan, "Study on the technology of trefoil polyamide 6 fiber," Modern Silk Science and Technology, vol. 27, no. 5, p. 173, 2012.

[11] R. G. Ling, C. E. Li, C. Y. Guo, L. D. Ma, and G. H. Xu, "Preparation of oxygen anionic PA6 profiled fiber," Journal of Silk, vol. 5, p. 35, 2010.

[12] K. Chen and X. D. Zhou, "Super-hydrophilic polyurethane modified block silicone M-588A," Journal of Silk, vol. 42, no. 23, p. 38, 2016.

[13] Y. T. Li, C. Tang, L. F. Zhang, and Y. L. Wang, "Synthesis and performance research of hydrophilic resin for coating," Guangdong Chemical Industry, vol. 13, no. 41, p. 49, 2014.

[14] J. Y. Hong, "Preparation and application of modified polyamide multifunction finishing agent for nylon fabric," Zhejiang Sci-Tech University, Hangzhou, China, M.S. thesis pp. 9-10, 2015.

[15] D. Pappas, A. Bujanda, J. D. Demaree et al., "Surface modification of polyamide fibers and films using atmospheric plasmas," Surface and Coatings Technology, vol. 201, no. 7, pp. 4384-4388, 2006.

[16] Y. Miao, Z. Ye, W. Xu et al., "p-Type conduction in phosphorus-doped $\mathrm{ZnO}$ thin films by MOCVD and thermal activation of the dopant," Applied Surface Science, vol. 252, no. 22, pp. 7953-7956, 2006.

[17] K. K. Samanta, M. Jassal, and A. K. Agrawal, "Improvement in water and oil absorbency of textile substrate by atmospheric pressure cold plasma treatment," Surface and Coatings Technology, vol. 203, no. 10-11, p. 1336, 2009.

[18] Y. Yan, Y.-H. Huang, Y. Wang, Z.-C. Xiao, and M.-B. Yang, "Polyamide 6 (PA6)/polyethylene terephthalate (PET) blends with gradient and encapsulation structure developed by injection molding," Polymer, vol. 180, p. 121679, 2019.

[19] N. Chen, X. Yao, C. Zheng et al., "Study on the miscibility, crystallization and crystalline morphology of polyamide-6/ polyvinylidene fluoride blends," Polymer, vol. 124, pp. 30-40, 2017.

[20] S. S. Banerjee, U. Gohs, C. Zschech, and G. Heinrich, “Design and properties of high-performance polyamide 6/fluoroelastomer blends by electron-induced reactive processing," European Polymer Journal, vol. 85, pp. 508-518, 2016.
[21] M. Han, "Thermodynamic and rheological variation in polysulfone solution by PVP and its effect in the preparation of phase inversion membrane," Journal of Membrane Science, vol. 202, no. 1-2, p. 55, 2002.

[22] W. Zhao, Y. Su, C. Li, Q. Shi, X. Ning, and Z. Jiang, "Fabrication of antifouling polyethersulfone ultrafiltration membranes using Pluronic F127 as both surface modifier and poreforming agent," Journal of Membrane Science, vol. 318, no. 12, p. 405, 2008.

[23] J. I. Clodt, S. Rangou, A. Schröder et al., "Carbohydrates as additives for the formation of isoporous PS-b-P4VP diblock copolymer membranes," Macromolecular Rapid Communications, vol. 34, no. 2, pp. 190-194, 2012.

[24] C. Yang, G. Zhu, Z. Yi, Y. Qiu, L. Liu, and C. Gao, "Tailoring the pore size and permeability of isoporous membranes through blending with poly (ethylene glycol): Toward the balance of macro-and microphase separation," Journal of Membrane Science, vol. 598, p. 117755, 2019.

[25] A. Idris, N. Mat Zain, and M. Y. Noordin, "Synthesis, characterization and performance of asymmetric polyethersulfone (PES) ultrafiltration membranes with polyethylene glycol of different molecular weights as additives," Desalination, vol. 207, no. 1-3, p. 324, 2007.

[26] J.-H. Kim and K.-H. Lee, "Effect of PEG additive on membrane formation by phase inversion," Journal of Membrane Science, vol. 138, no. 2, pp. 153-163, 1998.

[27] H. Huang, L. Yan, Y. Guo, H. L. Lin, L. Chen, and L. F. Yang, Journal of Polymer, vol. 188, p. 122119, 2019.

[28] S. Yu, K. H. Oh, and S. H. Hong, "Effects of silanization and modification treatments on the stiffness and toughness of BF/ SEBS/PA6, 6 hybrid composites," Composites Part B: Engineering, vol. 173, p. 106922, 2019.

[29] D. R. Dreyer, S. Park, C. W. Bielawski, and R. S. Ruoff, "The chemistry of graphene oxide," Chemical Society Reviews, vol. 39, no. 1, pp. 228-240, 2010.

[30] S. Zhang, P. Xiong, X. Yang, and X. Wang, "Novel PEG functionalized graphene nanosheets: enhancement of dispersibility and thermal stability," Nanoscale, vol. 3, no. 5, p. 2169, 2011.

[31] A. L. LV and C. Z. Chuai, "Synthesis and properties study of PA6-PEG copolymer," China Plastics Industry, vol. 42, no. 7, p. 11, 2014.

[32] B. D' Alò, G. Coppola, and B. Pallesi, "Studies of crystalline forms of nylon-6 by X-ray and i.r. spectrophotometry," Polymer, vol. 15, no. 3, p. 130, 1974.

[33] Y. Li and W. A. Goddard, "Nylon 6 crystal structures, folds, and lamellae from theory," Macromolecules, vol. 35, no. 22, pp. 8440-8455, 2002. 